\title{
Experiencia del curso de Práctica Profesional Supervisada de la carrera de Orientación desde la presencialidad remota, Universidad Nacional, Costa Rica durante el año 2020
}

Experience of the Supervised Professional Practice course of the Guidance
major, in remote modality, National University, Costa Rica during the year 2020

Experiência do curso de prática profissional supervisionada da carreira de Orientação a distância, Universidade Nacional, Costa Rica durante o ano de 2020

\author{
German González-Sandoval \\ Universidad Nacional \\ Heredia, Costa Rica \\ german.gonzalez.sandoval@una.cr \\ (1) ORCID: https://orcid.org/0000-0002-9149-7635
}

Recibido - Received - Recibido: 26 / 05 / 2021 Corregido - Revised - Revisado: 11 / 10 / 2021 Aceptado - Accepted - Aprobado: 29 / 10 / 2021

DOI: https://doi.org/10.22458/ie.v23i35.3559

URL: https://revistas.uned.ac.cr/index.php/innovaciones/article/view/3559

\begin{abstract}
Resumen: En el marco de la pandemia provocada por el virus responsable de la COVID-19, se presentan innumerables cambios a nivel global ante una realidad emergente; por lo tanto, la enseñanza superior asume el reto de transformar las metodologías tradicionales. Ante ello, se propone como objetivo de esta sistematización analizar las modificaciones metodológicas y evaluativas en el curso Práctica Profesional Supervisada de la carrera de Orientación en el 2020, desarrollado en la modalidad de presencialidad remota. El análisis se visualiza desde el rol de la persona académica a cargo del curso y los rubros correspondientes a la evaluación, lo cual visibiliza que la modalidad presencial remota incidió en la implementación de nuevas habilidades, tanto del profesorado en la planificación de las sesiones de clase como para las personas estudiantes al realizar su práctica; por ello, se evidencia una práctica planeada y ejecutada desde la realidad contextual, aspecto que genera mayor vínculo del estudiantado con su quehacer. Como principal conclusión se detalla que la modalidad de presencialidad remota en la Práctica Profesional Supervisada se debe mantener para el 2021; pues no resultaría conveniente exponer al estudiantado a riesgos de salud, traslado de residencia, cambios de trabajo y readaptaciones en el ámbito personal y familiar; además, desde la experiencia estudiantil y docente sobresalen resultados positivos de la ejecución desde esta modalidad.
\end{abstract}

Palabras clave: orientación pedagógica, enseñanza superior, estudiante universitario, pandemia, presencialidad remota, educación.

\begin{abstract}
Summary: In the context of the pandemic caused by the virus responsible for COVID-19, there are innumerable changes at a global level in the face of an emerging reality. Therefore, higher education takes on the challenge of transforming traditional methodologies. Given this, the objective of this systematization is to analyze the methodological and assessment modifications in the Supervised Professional Practice course of the Guidance major in 2020, developed in a remote modality. The analysis is viewed from the role of the academic person in charge of the course and the items corresponding to the evaluation, which shows that the remote face-to-face modality influenced the implementation of new skills, both of the teaching staff in the planning of the class sessions as well as for students when doing their practice. For this reason, a practice planned and executed from the contextual reality is evidenced, an aspect that generates a greater bond of the students with their work. The main conclusion is that the remote modality in Supervised Professional Practice must be maintained by 2021, since it would not be convenient to expose the student body to health risks, relocation of residence, job changes and readjustments in the personal and family sphere. In addition, from the student and teaching experience, positive results of the execution from this modality stand out.
\end{abstract}

Key Words: pedagogical guidance, higher education, university student, pandemic, remote presence, education.

Resumo: Experiência do curso de prática profissional supervisionada da carreira de Orientação a distância, Universidade Nacional, Costa Rica durante o ano de 2020. 
No contexto da pandemia causada pelo vírus responsável pela COVID-19, se apresenta inúmeras mudanças em nível global diante de uma realidade emergente; portanto, o ensino superior está assumindo o desafio de transformar as metodologias tradicionais. Em vista disto, o objetivo desta sistematização é analisar as modificações metodológicas e avaliativas no curso de Prática Profissional Supervisionada da carreira de Orientação em 2020, desenvolvido na modalidade de sala de aula remota. A análise é visualizada a partir do papel do acadêmico responsável pelo curso e dos itens correspondentes à avaliação, o que mostra que a modalidade presencial remota teve um impacto na implementação de novas habilidades, tanto para o corpo docente no planejamento das sessões de aula como para os alunos ao realizarem sua prática; portanto, evidencia uma prática planejada e executada a partir da realidade contextual, aspecto que gera um maior vínculo entre os alunos e seu trabalho. A principal conclusão é que a modalidade de presença remota na Prática Profissional Supervisada deve ser mantida para 2021; pois não seria conveniente expor os estudantes a riscos de saúde, transferência de residência, mudanças de emprego e reajustes no ambiente pessoal e familiar; além disso, a partir da experiência do estudante e do professor, destacam-se os resultados positivos da execução desta modalidade.

Palavras-chave: orientação pedagógica, educação superior, estudante universitário, pandemia, presença remota, educação.

\section{INTRODUCCIÓN}

El tema que ha propiciado la elaboración de esta sistematización es la práctica profesional supervisada, una actividad que representa un proceso de trabajo por parte de la persona estudiante y docentes en diversas carreras de enseñanza superior, y al respecto, se puede contextualizar con Peña y Vargas (2020) que:

El caso de Costa Rica es muy similar al de la República Mexicana, no existen leyes que regulen este tipo de actividades curriculares, cada universidad establece su propio reglamento para el cumplimiento de la práctica profesional, quienes, a su vez, reportan a los organismos y Oficinas de Transferencia de Conocimiento y Vinculación Externa del país, estos son los encargados de promover el desarrollo en atención a las necesidades de la sociedad y el potencial de oferta de la población universitaria, procurando estimular la innovación del conocimiento y fortaleciendo la vinculación externa por medio de la transferencia de conocimiento, que es lo que se desarrolla durante el proceso de práctica profesional. (p.60)

En este sentido, la Carrera de Orientación en la Universidad Nacional (UNA), en el nivel de Bachillerato tiene como requisito la realización de una actividad académica denominada Práctica Profesional Supervisada en Orientación (PPSO), la cual implica la sumatoria de un total de 195 horas en algún escenario laboral y disciplinario en una institución u organización gubernamental o no gubernamental, en donde se puedan desplegar acciones propias de la función disciplinaria y la persona estudiante u orientadora en formación desarrolle su trabajo de práctica, esto le permite reforzar tanto conocimientos teóricos como metodológicos; su importancia se conceptualiza desde la Universidad Nacional (2017), indicando que:

Es una experiencia que realiza el estudiante en un ámbito específico de aplicación de su especialidad disciplinaria o profesional, y a su vez, un espacio de aprendizaje que posibilita su proyección social y el establecimiento de relaciones teóricas, metodológicas y técnicas con la realidad. Todo lo cual concurre a la formación integral del estudiante. (p.45)

Esta Práctica Profesional Supervisada, se compone de tres actividades académicas: el curso de diseño de proyecto de PPSO que se genera en el I Ciclo, el curso de PPSO y la PPSO que se desarrolla complementaria y paralelamente en el II Ciclo. Al ser un requisito de graduación para el estudiantado, esta etapa comprende una de las actividades de mayor relevancia durante su formación universitaria. 
Por lo anterior, se debe reconocer que las actividades académicas del II Ciclo están insoslayablemente vinculadas e integradas tanto en su ejecución como en su evaluación. Gómez, Montes, Ortega, Salas y Villalobos (comunicación personal, octubre, 28, 2019) mencionan que la PPSO es la actividad que permite a la persona estudiante tener un acercamiento al contexto laboral de la Disciplina, y por otro lado, el curso por medio de sesiones teóricas nutre el conocimiento adquirido el proceso de formación, así como su actuar en la PPSO como tal.

La ejecución de la Práctica Profesional Supervisada la conduce una persona académica supervisora, quien debe brindar acompañamiento profesional y supervisión a un aproximado de ocho estudiantes, con los cuales se reúne semanalmente para dar seguimiento a las acciones de su proyecto de PPSO, por medio de la evaluación de rubros como entrevistas, sesiones colectivas, estudio de caso, informe final del proyecto, entre otros, que en total corresponden a un $90 \%$ de un $100 \%$ en total, porcentaje que hasta el 2019 se mantuvo de esa manera.

Por otra parte, el Curso de Práctica Profesional Supervisada es mediado por la persona académica responsable del curso, quien evalúa el $10 \%$ restante, por medio de la asistencia de las personas estudiantes a las sesiones de clase semanales, destinadas a la ejecución de talleres, charlas y conferencias por parte de personas profesionales invitadas.

Como se puede inferir, el curso tiene la finalidad de promover el fortalecimiento de habilidades conceptuales, procedimentales y actitudinales para el desarrollo del proyecto de PPSO. En cuanto al desarrollo de este curso a nivel histórico, se contempla de manera integrada tanto el grupo 16 (horario matutino) como el grupo 19 (horario nocturno), juntos en un solo grupo y en el horario nocturno que inicia a las 17 horas, en la modalidad presencial.

De lo anterior expuesto, se puede analizar que la evaluación recae en la persona académica supervisora de PPSO, por otro lado, la participación de la persona académica a cargo del curso se limita a la evaluación de la asistencia, lo cual impide generar procesos valorativos en relación con la evolución de los procesos fundamentales en el ejercicio de la Orientación, como la toma de decisiones, liderazgo, trabajo en equipo, resolución de problemas y la capacidad para la vinculación, coordinación y gestión, relacionada a la labor orientadora que establece el descriptor del curso.

Lo antes expuesto refleja la normalidad en la que transcurrían estos procesos académicos antes de la pandemia suscitada en el 2020; sin embargo, con la aparición del virus responsable de la COVID-19, se comunica mediante el Decreto Ejecutivo 42227-MP-S, el 16 de marzo de 2020 firmado por el Presidente de la República, el Ministro de Salud y la Ministra a.i. de la Presidencia, la declaratoria de Estado de Emergencia Nacional por efecto de la pandemia y el establecimiento de normas sanitarias a nivel nacional.

En consecuencia, se hizo necesaria la aplicación de nuevos términos; tal como la educación a distancia "[...] que puede darse mediante el uso de plataformas que permitan el acceso a aulas virtuales, que faciliten tanto el encuentro de alumnos y profesores, como el acceso a materiales educativos y de enseñanza en formato de texto y audiovisual" (CAF-CEPAL, 2020, p.22). De esta manera, el Consejo Académico de la UNA (2020) definió algunas normas para la atención de su comunidad institucional; la determinación más significativa es el cambio de clases de la modalidad presencial a modalidad de presencialidad remota, definida como la alternativa de participación sin límite geográfico en procesos educativos de manera sincrónica o asincrónica.

Lo anterior implicó que las autoridades de la División de Educación para el Trabajo (DET) y los responsables del Proyecto denominado "Actividad Académica Permanente Comisión de Práctica Profesional en Orientación" (Proyecto encargado de gestionar y coordinar las diversas acciones a nivel institucional e interinstitucional) a cuestionarse y valorar las posibilidades de suspender-como lo realizaron en otras Unidades Académicas- o bien, ajustar la PPSO. 
Con la finalidad de proteger a la población estudiantil de la interacción con otras personas donde se podrían exponer a riesgos de contagio ante el virus responsable de la COVID-19, las autoridades de la Unidad Académica determinaron la realización de una serie de ajustes que permitieran la puesta en marcha de la actividad de PPSO durante el 2020 y con ello favorecer la eventual finalización del Bachillerato Universitario de la comunidad estudiantil.

Sobre esta línea, se puede garantizar la permanencia de la acción sustantiva de la UNA, mediante el desarrollo de la docencia universitaria apoyado en que:

[...] siendo el reto y responsabilidad de la docencia universitaria no tanto "enseñar" los conocimientos de una ciencia sino "EXPLICARLOS" desde dicha ciencia, la formación del estudiante universitario no es aprender los conocimientos de una ciencia, sino "aprender a pensar" dichos conocimientos desde dicha ciencia. Ahora bien, la única manera de "enseñar a pensar" una ciencia consiste en EXPLICAR Y COMPRENDER sus conocimientos. (Sánchez, 2003, p.22)

En contexto, se puede afirmar que la docencia en sí misma no cambia, sino que ese reto y responsabilidad del que habla el autor se asume con el cambio en la metodología, evaluación y espacio de encuentro con el colectivo estudiantil; pues al ajustarse a los cambios suscitados, el espacio de interacción se ejecutará mediante una reunión presencial remota. Además, Cañedo y Figueroa (2013) citando a UAA, mencionan que la persona docente universitaria debe:

[...] usar estrategias metodológicas que faciliten el aprendizaje, monitorear, retroalimentar dicho proceso y adaptar lo que enseña a los ritmos y necesidades de los estudiantes, además de favorecer el desarrollo de habilidades para el trabajo independiente, brindarles oportunidades para aprender fuera de la institución, y desarrollar en ellos una autoestima positiva. Asimismo, el profesor deberá asumir su actividad académica como una carrera de vida y usar los recursos a su alcance para mejorar su capacidad profesional y poseer una actitud crítica que le permita hacer aportaciones a la institución a fin de mejorar el ambiente laboral y académico (p.26)

Esos cinco elementos señalados en la cita son aspectos que no se pueden dejar de lado en el entorno docente actual; pues la autogestión estudiantil es un elemento vital, tanto para alcanzar los objetivos académicos como para favorecer su crecimiento personal. Asimismo, para comprender la docencia universitaria es necesario visualizar más allá del acto meramente educativo y trascender en la transmisión de valores y actitudes del ser profesional, así como promover un ambiente saludable que garantice las interacciones, ya sean físicas o virtuales en la comunidad universitaria. Al respecto, Hodges, Moore, Lockee, Trust, y Bond (2020) mencionan algunos factores a tomar en cuenta en la implementación de clases bajo la presencialidad remota, y apuntan que:

Thus, careful planning for online learning includes not just identifying the content to cover but also carefully tending to how you're going to support different types of interactions that are important to the learning process. This approach recognizes learning as both a social and a cognitive process, not merely a matter of information transmission.

[la planificación cuidadosa del aprendizaje en línea incluye no solo identificar el contenido que se debe cubrir, sino también prestar atención a cómo apoyar a los diferentes tipos de interacciones que son importantes para el proceso de aprendizaje. Este enfoque reconoce el aprendizaje como un proceso social y cognitivo, no simplemente como una cuestión de transmisión de información]. (párr. 11) 
De estos mismos autores es necesario resaltar que no es lo mismo educación en línea que la enseñanza remota a causa de la emergencia, debido a que el primero está planeado desde un principio con esa finalidad; mientras el segundo surge a partir de las adaptaciones que forzosamente se hicieron a causa del obligatorio distanciamiento físico. Sin embargo, se realizan grandes esfuerzos por procurar la misma calidad, educación oportuna y efectividad como si los elementos que componen el curso estuviesen planeados para su aplicación en línea.

En este sentido, para comentar de esa docencia en la virtualidad que es favorecedora del aprendizaje significativo de las personas estudiantes, Moreira y Delgadillo (2014), realizan la siguiente indicación al respecto:

[...] para que se produzca un verdadero aprendizaje, es decir, un aprendizaje a largo plazo que no sea sometido al olvido, es necesario conectar los conocimientos nuevos con los previos, por lo que se hace imprescindible presentar estos conocimientos al alumno de manera coherente y no arbitraria, "construyendo" de manera sólida los conceptos, interrelacionándolos unos con otros en forma de red de conocimiento. La función del docente es engarzar los procesos de construcción del alumno con el saber colectivo culturalmente organizado y en relación directa con su contexto social. Por eso se insiste en formar al estudiante, que va a verse inmerso en este contexto, y cuánto más al profesional que va a formarse. (p.125)

Aunado a lo expuesto anteriormente, cabe señalar en este punto, que las orientaciones epistemológicas tanto del presente artículo, así como lo planeado y ejecutado en el curso de PPSO se sustenta en el modelo constructivista o socio-constructivista, según el autor que se tome en cuenta. De esta manera, se puede señalar que el socio-constructivismo es la posición en donde el conocimiento es:

[...] el resultado de un proceso de construcción o reconstrucción de la realidad que tiene su origen en la interacción entre las personas y el mundo [...] está en la mente de las personas y que el sujeto cognoscente no tiene otra alternativa que construir lo que conoce sobre la base de su experiencia. El conocimiento entonces es construido a partir de las experiencias individuales, aunque también existe el conocimiento colectivo que se ubica en los grupos culturales. (Camacho, Átala, Maldonado, Álvarez, Flores y Santander, 2011, párr. 4)

Adicionalmente, es necesario sustentar las decisiones de los cambios que se proponen para que la evaluación se genere de la manera más oportuna y consiente del contexto en el que se ejecuta el curso; por esto se toma en cuenta que "las actividades evaluativas constructivistas propician la evaluación auténtica, que se refiere a la aplicación práctica, a la vida real, de los conocimientos, habilidades, competencias o actitudes que se han construido en el proceso de aprendizaje" (Salgado, 2006, p.86). Para sustentar este aporte de Moreno (2009) se retoma que:

Actualmente se tiende a una evaluación integrada de manera natural en el proceso didáctico que abarque al alumno como sujeto que está aprendiendo, globalizadora de toda su personalidad, reconociendo que este conocimiento holístico demanda comunicación abierta con él, comprender sus problemas, circunstancias, su trabajo escolar; en definitiva, asumir una postura humanista sobre la educación (p.568)

Estos constructos están orientados hacia la enseñanza superior tradicional de presencialidad; no obstante, de manera imperante deben plasmarse en la docencia universitaria practicada desde la presencialidad remota, pues en estos tiempos resalta la necesidad de un enfoque humanista e integral, 
en el que se consideren las experiencias y los contextos tan diversos que se viven al enfrentarse a un mundo cambiante en el ámbito laboral, económico, cultural, social, entre otros.

Según la realidad expuesta, el reto generado, las posiciones teóricas y epistemológicas, este artículo pretende analizar las modificaciones metodológicas y evaluativas en el curso Práctica Profesional Supervisada de la carrera de Orientación en el 2020, desarrollado en la presencialidad remota.

\section{DESARROLLO DE LA EXPERIENCIA}

Si se parte de lo expuesto por Sánchez (2010), en cuanto establece que "[...] la sistematización de experiencias está vinculada con la metodología Investigación Acción Participativa (IAP), dado que una de sus características es que, a partir de reflexiones colectivas, los involucrados se auto-investigan para comprender y generar activamente cambios institucionales y/o sociales" (p.5); en este caso, la población participante involucrada se compone de las 51personas matriculadas en el curso de PPSO, debido a que fueron protagonistas de la experiencia; se les consultó por medio de un formulario de Google, un informe final y sesión sincrónica de evaluación general de los webinarios y, por último, una evaluación del curso mediante una sesión sincrónica.

La súbita, aunque necesaria adaptación de las actividades académicas en la UNA provocó la definición de nuevas disposiciones generales para la PPSO 2020, de las cuales sobresalen las relacionadas con la redistribución de los rubros de evaluación; pues se asignan más elementos por evaluar a la persona académica responsable del curso de PPSO. (Actividad Académica Permanente de PPSO, comunicación personal, julio, 15, 2020).

El rol de la persona académica responsable del curso se mantiene tanto en su finalidad como en el desarrollo del curso, e integra asimismo a ambos grupos; sin embargo, en el 2020 se redefine la evaluación, pasa de un $10 \%$ a un $30 \%$. Por tal motivo, en el diseño del curso se deben integrar los rubros de las sesiones de clase en la modalidad de presencialidad remota mediante la metodología de los webinarios, la elaboración del aporte a la comunidad y la asistencia y participación.

Para el diseño del programa del curso, la persona académica responsable define procesos evaluativos iniciales, de proceso y finales, donde se toma en cuenta lo expuesto por García, Corell, Abella, y Grande (2020), quienes apoyados en Boud y Falchikov, y Carless, mencionan que la evaluación que se orienta al aprendizaje de la persona estudiante contempla tres aspectos medulares: tareas que realiza el estudiantado, retroalimentación de la persona docente y la participación de ambas partes en aspectos evaluativos. Esta tarea académica implica la valoración contextual para la ejecución y el desarrollo, que se fortalece con la evaluación del proceso y final, tal y como lo plantea González (2020):

El nombre de ADDIE se obtiene del acrónimo en inglés de las diferentes partes que conforman a este modelo: análisis, diseño, desarrollo, implementación y evaluación. Describiré brevemente cada etapa: 1) Análisis: se evalúa quiénes serán los estudiantes, los contenidos a enseñar y el entorno en el que se desenvolverá el curso. 2) Diseño: se prepara el contenido del curso a impartir, se plantea el enfoque didáctico con el que se impartirá el curso, se generan actividades e instrumentos de evaluación, se preparan las actividades de aprendizaje a llevar a cabo y se definen los recursos que se utilizarán. 3) Desarrollo: se realiza la implementación de los recursos que se utilizarán en las plataformas educativas o las páginas web; se elaboran y prueban. 4) Implementación: se da la interacción con los alumnos y se realizan propiamente las actividades de aprendizaje. 5) Evaluación: se valoran los resultados obtenidos en el curso, así como los procesos y los recursos de enseñanzaaprendizaje. Además, se puede evaluar cada fase, con lo cual se pueden hacer ajustes cuando sea necesario (p.16) 
Por la modalidad de presencialidad remota se modifican las sesiones de clase semanales, las cuales se utilizarán para profundizar los temas valorados como prioritarios para el grupo y mediante la estrategia metodológica de webinario, conceptualizada por Zielińsk, Jaruga, Hofmann, Marinova, Plewczynski, Kerler (2012) como "Web-based seminar with transmission of video and audio content online (over the internet) from one source to a limited audience with the purpose of training". [Seminario basado en la web con transmisión de contenido de video y audio en línea (a través de Internet) desde una fuente a una audiencia limitada con el propósito de capacitación]. (p.10)

Sobre las virtudes que se anteponen a otras estrategias metodológicas virtuales, el webinario tiene la posibilidad de interacción y tránsito de información fluida tal y como resaltan Zielińsk et al., (2012):

A key feature of a good webinar is its interactive elements - the ability to give, receive and discuss information. An interactive function can be fulfilled by discussion boards and online chat-rooms provided on the same website as the webinar. This either allows trainees to discuss the content as an online community or, in some cases of live discussion and panel shows, contribute to the presentation directly by asking questions or making statements which can be relayed directly to the panel. As the trainees are given an increased level of interactivity by allowing them to submit information to the discussion (be it text, graphic, audio or video) the experience will become more engaging for them and make for abetter learning experience.

[Una característica clave de un buen seminario web son sus elementos interactivos: la capacidad de dar, recibir y discutir información. Los foros de discusión y las salas de chat en línea que se ofrecen en el mismo sitio web que el seminario web pueden cumplir una función interactiva. Esto permite a los participantes discutir el contenido como una comunidad en línea o, en algunos casos de discusiones en vivo y programas de panel, contribuir a la presentación directamente haciendo preguntas o haciendo declaraciones que se pueden transmitir directamente al panel. A medida que los alumnos obtienen un mayor nivel de interactividad al permitirles enviar información a la discusión (ya sea texto, gráfico, audio o video), la experiencia se volverá más atractiva para ellos y mejorará la experiencia de aprendizaje]. (p.10)

Este ajuste se realiza con el objetivo de favorecer el compartir de experiencias y conocimientos que aportan las personas profesionales invitadas, lo cual tiene estrecho vínculo con la posición epistemológica socio-constructivista, esto favorece el protagonismo del colectivo estudiantil en el desarrollo de los webinarios; pues se asignan dos temas para cada subgrupo, quienes planifican su desarrollo, ejecución y evaluación -esto contempla la organización total de dicha actividad, la misma con una duración de una hora y treinta minutos, dirigida al colectivo de personas de la Práctica Profesional Supervisada, estudiantes, académico responsable del curso, personas académicas supervisoras e invitadas-.

De esta manera, las temáticas por abordar en los webinarios se definen mediante una consulta por medio de un "Formulario de Google", con la finalidad de realizar un diagnóstico inicial, con el cual se pretende conocer sobre la perspectiva de las necesidades formativas del colectivo estudiantil, estas se agrupan en un listado de relación y pertinencia de unos temas con otros, en el caso particular se logró determinar los siguientes:

- Familia

- Necesidades educativas

- Área de orientación vocacional

- $\quad$ Orientación en Centros Penitenciarios

- TREC y Análisis transaccional

- Marco ético y jurídico de Orientación

- TICS en la Orientación

- Prevención y atención de adicciones

- RAC

- Procesos de duelo

- Empleabilidad y emprendimiento 
De acuerdo con lo expuesto por Zielińsk, K., et al., (2012), los beneficios de los webinarios radican en que se logra transmitir contenido y se capacita por medio de video y audio, lo cual permite una mayor interacción en el proceso de dar y recibir información, estas se pueden enriquecer por medio de salas de discusión, el chat de la plataforma entre otras.

Con este fundamento como apoyo, cada subgrupo responsable de la planeación y la ejecución del webinario, también fue responsable de realizar un informe final que contempla una autoevaluación, adicionalmente, una vez ejecutadas todos los webinarios, se realizó una sesión sincrónica en la que se efectuó una evaluación general por parte de estudiantes y el académico responsable del curso. De dichas evaluaciones surgen criterios valiosos situados en tres momentos específicos: antes, durante y después de la ejecución; de estos se pueden extraer los siguientes aspectos:

- Se le asigna importancia al acompañamiento, supervisión y apoyo de las personas académicas vinculadas al proceso de PPSO; pues como se menciona: "el apoyo de la profesora supervisora fue vital para el desarrollo de la Webinar ya que ella nos apoyó en todo momento y nos brindó gran acompañamiento durante todo el proceso". (Comunicación personal, Vetsay Quesada, setiembre, 2020)

- La selección oportuna de profesionales invitados para los webinarios se requiere para el éxito de estrategias, tal como se observa en la siguiente opinión de una estudiante: "la energía y personalidad de este facilitador ayudó mucho a que este webinar fuera un éxito para nosotros como grupo expositor y para todo el grupo en general como Orientadoras y Orientadores en formación". (Comunicación personal, Llobelky Hasbun, setiembre, 2020)

- Le conceden importancia a la planificación anticipada; pues permite la visualización integral de la experiencia, ya que "la planificación y organización previa con la que se cuenta, cada compañera encargada fue apta para el desarrollo de los espacios en el webinar". (Comunicación personal, Llobelky Hasbun, octubre, 2020)

- Realizar la coordinación previa con el profesional invitado pues se debe conocer la pertinencia de la temática que se abordará, lo cual se resalta en el siguiente comentario: "el contacto de la profesional, quien cuenta con una gran preparación académica, fue vital para que este seminario tuviera un impacto significativo en la población participe e incrementara su aprendizaje en la preparación profesional". (Comunicación personal, Allan Arias, septiembre, 2020)

- La mayoría de los temas abordados sí fueron de gran utilidad para un futuro laboral y estos lograron llamar la atención en las personas practicantes y puntualizan que "[...] la sección de preguntas estuvo favorablemente cargada, lo que motivó al profesional a profundizar en sus respuestas". (Comunicación personal, Anónimo, octubre, 2020)

- Sobre la organización de los webinarios se expresan sentimientos de satisfacción, tanto por el compromiso de sus compañeros y compañeras, aspecto que se refleja en la siguiente apreciación: "el trabajo en equipo fue gratificante, el apoyo entre todos y todas generó la finalización del webinar, una satisfacción de la experiencia". (Comunicación personal, María Isabel Porras, setiembre, 2020)

- Una persona estudiante de manera implícita se refiere a la situación de emergencia nacional por los motivos de la pandemia, específicamente señala: "este fue un trabajo que me lleno que orgullo ya que a pesar de la situación que estamos enfrentando se puede lograr un buen trabajo en equipo y además un Webinar muy exitoso". (Comunicación personal, Alexa Alfaro, setiembre, 2020)

Por otra parte, el aporte a la comunidad se concebía como un insumo que entrega la persona estudiante a la institución en la que realizó su Práctica; con el objetivo de favorecer el compromiso social y las habilidades de gestión para conseguir recursos materiales que requiera dicha institución. Sin embargo, 
en el 2020, tal insumo se ha replanteado como un aporte disciplinario con la finalidad de contribuir en un proceso orientador ya sea de divulgación, sensibilización o intervención, en el cual se plasme su utilidad, el respaldo teórico y, en caso de ser requerido, su guía de ejecución, mediante la elaboración de material visual o audible, ya sea físico o digital (vídeo, infografía, manual, podcast, entre otros).

Se debe indicar que para el 2020 el "aporte a la comunidad" se enfoca en las capacidades y habilidades profesionales y personales; por lo tanto, la producción realizada benefició diversos ámbitos de manera directa e indirecta, todos ellos relacionados con la acción orientadora y en los escenarios laborales en donde se desarrolló la PPSO de 2020, a saber:

- Personas que se encuentran en las etapas de la niñez, adolescencia y adultez que son estudiantes de Jardines de niños/niñas, escuelas, colegios y universidades.

- Comunidades educativas de diversas instituciones (padres y madres de familia, encargados, docentes, personas profesionales en orientación y otras disciplinas).

- Personas adultas mayores que asisten a Centros Diurnos.

La realización del aporte a la comunidad implicó una ardua tarea de investigación, planificación y diseño, pues las modificaciones metodológicas y evaluativas en el curso favorecieron el desarrollo de habilidades y competencias laborales, así como lo menciona Berger y Frey, citados por la Comisión Económica para América Latina y el Caribe (CEPAL)/Organización de Estados Iberoamericanos para la Educación, la Ciencia y la Cultura (OEI), (2020):

La combinación de habilidades para favorecer la adaptación a los cambios y tendencias tecnológicas es la clave para la formación del alumnado. Por lo tanto, la educación debe centrarse en impartir "habilidades de fusión": combinación de habilidades creativas, empresariales y técnicas que permiten a los trabajadores cambiar a nuevas ocupaciones a medida que emergen. (p.45)

En cuanto al tipo de productos elaborados como insumo o aporte a las comunidades, se evidenció en la población estudiantil un adecuado conocimiento de los entornos en los cuales se vincularon durante sus prácticas, en sus aportes se visualiza la pertinencia y la correspondencia de sus diseños con las diversas realidades de esos aportes a las comunidades, al respecto se realizaron los siguientes: siete videos interactivos, seis infografías, dos revistas, un blog que consta de cinco carpetas y seis manuales de diversos diseños y modalidades, elaborados con distintas herramientas tecnológicas, sitios web, técnicas e instrumentos para favorecer la intervención desde la disciplina mediante el trabajo grupal e individual. 
En la figura 1 se puede observar, a modo de ejemplo, la capacidad creativa y profesional demostrada:

\section{FIGURA 1}

Ejemplos de aportes a la comunidad elaborados por las personas estudiantes del curso de PPSO.

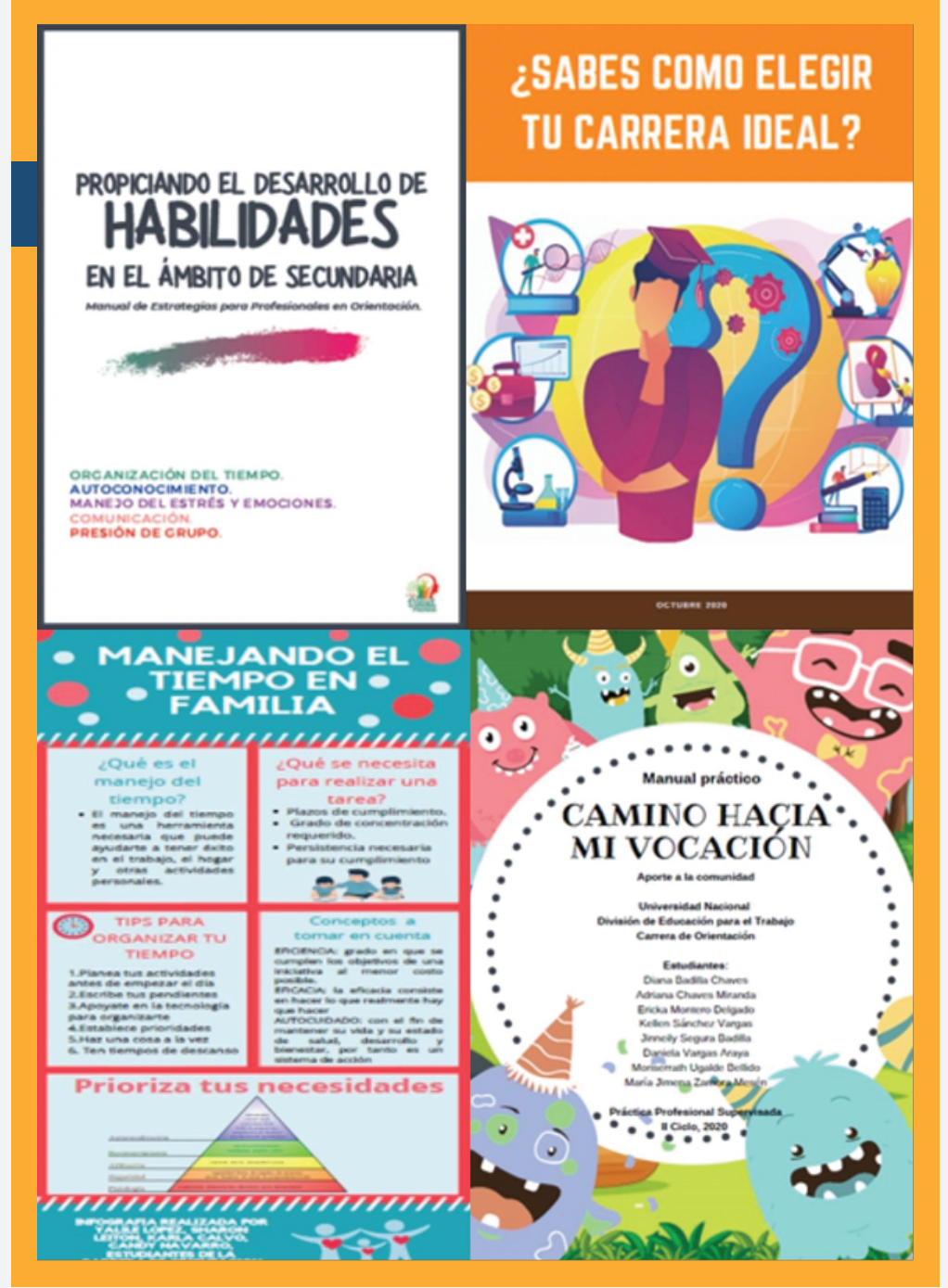

Fuente: Elaboración propia.

Por la pertinencia de los temas seleccionados resulta inevitable aportar el universo de temáticas y modalidades de intervención sugeridas por el estudiantado desde la PPSO, tal y como se observa a continuación:

- Ansiedad y estrés (conceptualización, síntomas, manifestaciones e identificación de manifestaciones físicas, cognitivas y psíquicas y recomendaciones para su prevención).

- Técnicas para el manejo del tiempo.

- Desarrollo vocacional y atención del área vocacional desde los diversos estilos de aprendizaje.

- Promoción de competencias para la educación emocional. 
- Educación emocional.

- Resiliencia.

- $\quad$ Situación de pandemia.

- Tips para la virtualidad.

- Promoción de habilidades para la vida:

- Organización del tiempo.

- Autoconocimiento.

- Manejo del estrés y emociones.

- Comunicación.

-Presión de grupo.

- La identidad y la satisfacción de necesidades del ser humano:

- La necesidad de amar y ser amado.

- La necesidad de sentirse útil o de mantener un nivel satisfactorio de conducta.

- Proceso vocacional y búsqueda de propósito en la persona, desde las cuatro componentes: hacer, pensar, sentir y su fisiología.

- Las redes sociales (importancia, conceptualización, principales usos y los protocolos para el adecuado funcionamiento).

- Manejo saludable del duelo (información general, exploración, reconocimiento de sentimientos y pensamientos frente al duelo y estrategias de afrontamiento).

- Duelo (tareas, etapas, tipos y abordaje).

- Promoción de la salud mental.

- Bienestar integral (área física, social, emocional, ética y vocacional).

- Relaciones intergeneracionales o convivencia entre familiares de diferentes etapas del ciclo vital.

Se visualiza que la población estudiantil asume un compromiso, un acercamiento auténtico y posición profesional para conocer a la población meta, a la vez que promueve la pertinencia y la relevancia de su práctica profesional.

Finalmente, respecto al rubro de evaluación de asistencia y participación, se debe mencionar que es obligatoria; por lo tanto, ante la modificación metodológica de presencialidad remota, implica que la persona estudiante deberá no solo asistir, sino participar activamente en cada una de las estrategias desarrolladas a lo largo del curso, esta rúbrica será valorada con un $10 \%$, el cual se alcanza con la participación a la totalidad de sesiones realizadas.

Además, su compromiso también se observa en la disposición para incorporar nuevos aprendizajes a través de las sesiones de clases virtuales, al tiempo que deja un excelente porcentaje de asistencia y participación en cada una de las actividades ejecutadas. No obstante, más allá de señalar que el curso de PPSO logró el 100 \% de promoción, se puede resaltar que efectivamente se dio una participación activa y fundamentada en cada una de las sesiones desarrolladas, y segundo que la asistencia fue un aspecto relevante; pues solo un 0,28 \% representa la inasistencia a las diversas actividades académicas efectuadas. No se presentó abandono ni reprobación, ni tampoco situaciones negativas en torno a la 
dinámica propia del curso, sobresale el desarrollo de habilidades diversas, entre ellas: tecnológicas, habilidades prácticas y disciplinarias.

La excelente asistencia y participación permitió que se favoreciera la intención de las actividades curriculares, las cuales radicaron en la integración y la actualización; además se alcanzó el cumplimiento de los objetivos planteados, principalmente en lo referente a la potenciación de habilidades y competencias en la conducción de procesos de Orientación con las diversas poblaciones de la PPSO, así como la valoración de la realidad contextual en el entorno que permitió la pandemia.

De este curso se debe resaltar su proyección y compromiso con la sociedad costarricense por el nivel de aportes, tanto en el abordaje individual como grupal de diversas poblaciones $y$, en especial de aquellas que han sido reiterativamente más vulnerabilizadas; sin duda, la experiencia de PPSO es una que califica como Extensión Universitaria en sí misma.

Cabe resaltar que el último método de consulta utilizado se ejecutó mediante una sesión virtual sincrónica para la evaluación final respecto a las modificaciones metodológicas y evaluativas del curso de PPSO, esto se fundamentó en lo mencionado por Camacho, et al. (2011), con lo cual se reconoce que el conocimiento se construye a través de las experiencias de las personas estudiantes; para ello se transcriben las siguientes frases expresadas, a saber:

\section{FIGURA 2}

Frases expresadas por algunas personas estudiantes en la sesión de evaluación final del curso de PPSO 2021.

He aprendido de tantos temas pertinentes y a profundidad, ha sido enriquecedor...

Muy importantes y fundamentales, además se desarrollaron habilidades y herramientas para la elaboración de Webinar...

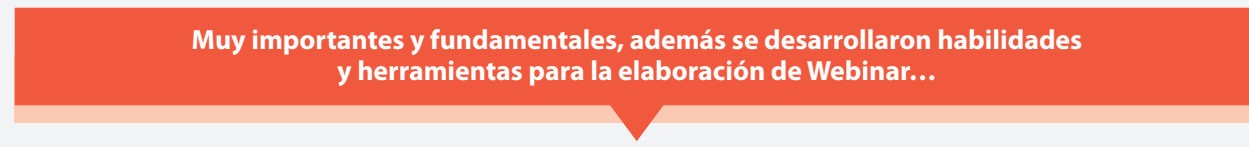

Webinar acertados y muy buenas temáticas, se debe regular la duración de los espacios y programar un receso de al menos 10 minutos...

Fundamental en especial por el uso de la tecnología para enfrentar la actualidad virtual y la enseñanza a distancia..

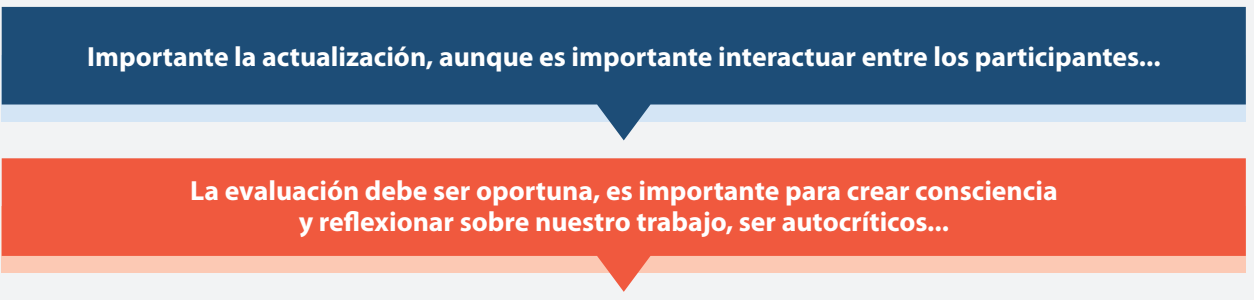

Que los participantes logren evaluar de manera consciente y comprometida...

Es necesario mantener siempre las cámaras encendidas...

Fuente: Elaboración propia. 
De la información anterior, se puede interpretar una percepción positiva del estudiantado acerca de la realización de las estrategias propuestas para el desarrollo del curso, entre ellas sobresale la estrategia del webinario y se hace el señalamiento de mejora de aspectos vinculados con la evaluación y los elementos técnicos de estos. En términos generales, es importante rescatar que, a pesar de las cargas académicas y las condiciones generadas por la presencialidad remota, se finaliza el curso con el cumplimiento de los objetivos propuestos en su programa, en un ambiente placentero y de satisfacción para las personas involucradas..

\section{SÍNTESIS Y REFLEXIONES FINALES}

Se han enumerado las experiencias y sus valoraciones como parte de un proceso permanente y participativo, por ello se hace necesario ponerlo en conocimiento de la comunidad académica y favorecer el tránsito de la información para promover los aprendizajes significativos y la colectividad del conocimiento.

En primer plano, este recorrido permite identificar, reconocer y visualizar las vivencias propias del curso desde el horizonte, aunque el reto es lograr una mirada lo más objetiva posible para interpretar, valorar y redefinir el diseño de una experiencia que definitivamente impregnará las acciones a desarrollar durante el 2021 en adelante.

Las condiciones de pandemia aún se mantienen vigentes en cuanto a los eventuales riesgos que podría afrontar el colectivo estudiantil en una modalidad totalmente presencial; por lo tanto, la información acá contenida se debería convertir en insumos para favorecer la discusión y la planificación de las autoridades de la Unidad Académica, las personas responsables de la Actividad Académica Permanente de PPSO 2021 y las personas académicas que se vinculen en los próximos procesos de PPSO.

En consecuencia, se hace meritorio resaltar algunas consideraciones extraídas de la experiencia docente con respecto al desarrollo de la PPSO, mediante la presencialidad remota y en atención a los ajustes requeridos para su ejecución, entre las cuales se señalan las siguientes:

- La atención de las condiciones de la pandemia ha implicado un "alto en el camino" para valorar la acción educativa y sus metodologías que, por muchos años, se mantuvo intocable y "estable".

- La modalidad de presencialidad remota generó primero la incertidumbre sobre la eventual suspensión de la Práctica Profesional Supervisada en Orientación durante el 2020 y, posteriormente, sobre el desarrollo de esta, según los nuevos lineamientos definidos por la Unidad Académica.

- El diseño del programa del curso requirió una adaptación en las estrategias evaluativas, tanto en su metodología como en sus valoraciones.

- Se presenta un aporte a la comunidad con una mayor elaboración disciplinaria por parte del estudiantado, pertinente y en congruencia con las necesidades de la comunidad en la que se desarrolló su PPSO.

- La gestión de dos webinarios para cada subgrupo de trabajo, implicó un esfuerzo de planificación grupal que favoreció el reconocimiento de habilidades y destrezas a nivel colectivo, así como capacidades de liderazgo y trabajo en equipo.

- Los cambios de metodología y evaluación del curso promueven las competencias tecnológicas en el estudiantado y las personas académicas, esto implica incluso la redefinición de sus roles de vinculación. 
- La participación activa de personas académicas en la experiencia de PPSO realizada en el 2020, logró consolidar aprendizajes significativos en el desarrollo de la PPSO en un contexto adverso y emergente que permitió la atención oportuna y la supervisión adecuada de la población estudiantil.

Sin duda, esta ha sido una práctica innovadora en la ejecución del curso de PPSO, por tal motivo es importante adentrar en las reflexiones que dejen en evidencia las experiencias vividas y los aprendizajes que se tuvieron antes, durante y después de su ejecución. En relación con los webinarios como estrategia de mediación pedagógica se valora las facilidades de participación, el desarrollo de estrategias para el abordaje teórico y práctico de los temas desarrollados.

Esta estrategia permitió un acercamiento con profesionales de Orientación, así como de otras disciplinas, quienes de alguna manera $u$ otra se han especializado o han desarrollado amplia experiencia entre los temas específicos, por ello se promovió el conocimiento de la realidad laboral y profesional de diversos ámbitos laborales, sin duda los temas abordados revisten de gran utilidad para el futuro laboral. Se debe rescatar que las participaciones en términos generales favorecieron la actualización y el refrescamiento de temas disciplinarios.

En relación con el trabajo de las y los estudiantes se debe rescatar la elaboración de materiales por su creatividad y utilidad para su desarrollo, el compromiso demostrado en la programación y el desarrollo de los diferentes webinarios, a pesar de la poca o nula experiencia en algunos casos; pues no habían tenido que explorar distintas plataformas virtuales de trabajo individual o grupal.

Ante los positivos comentarios y resultados, la gestión de experiencias como los webinarios para cada subgrupo de trabajo debe mantenerse y alternarse con actividades que impliquen, no solo el desarrollo teórico, conceptual y contextual, sino también el desarrollo de habilidades prácticas para el fortalecimiento del quehacer disciplinario, no sin antes obtener información de primera mano sobre las percepciones y valoraciones de la comunidad estudiantil y la persona académica responsable del curso de PPSO, para definir aspectos de mejora respecto a los aspectos metodológicos de evaluación y elementos técnicos propios del desarrollo de los webinarios.

Por otra parte, y con el fin de retomar algunos párrafos adelante expuestos, se mencionaba que históricamente el aporte a la comunidad tuvo por objetivo el desarrollo de capacidades de gestión en la persona estudiante, lo cual es necesario mencionar; pues tiene una gran relevancia para la labor diaria. Sin embargo, para el 2020 ese fin es repensado y se dirige hacia la promoción y el desarrollo de habilidades y capacidades tanto profesionales como personales; pues el estudiantado es retado profesionalmente a pensar en la manera de construir un aporte que sea significativo para la población, pero que además resulte un insumo relevante desde la disciplina de Orientación.

El hecho de que las personas estudiantes lograran preparar ese insumo, implicó la utilización de diferentes plataformas o aplicaciones virtuales que estuviesen a disposición para generar un producto no solo útil, sino también visual o auditivamente atractivo y congruente con el objetivo o temática planteada para su elaboración, garantizó un desarrollo de la acción orientadora pertinente y congruente con las necesidades de las comunidades y además contextualizado con el mundo virtual en donde se está inmerso, aún más ante la llegada de la pandemia y la obligada tarea de ocuparse desde la presencialidad remota.

De igual forma se debe señalar que la tarea de elaborar una propuesta pertinente para las poblaciones con las que desarrollaron sus prácticas profesionales, es una manera de demostrar su compromiso social entendido como la oportunidad para que la persona estudiante contribuya con las demandas de la sociedad en una comunidad específica $y$, de igual manera, elaborar propuestas que permitan el desarrollo de las personas con las cuales interactuaron de forma virtual durante el 2020, así como comprender que dejar un insumo trasciende el hecho de llegar a las comunidades y "servirnos de ellas" $y$ 
permite que la persona estudiante demuestre, con sus aportes, una forma de agradecer la oportunidad de favorecer su desarrollo y su formación profesional.

Por último, respecto a la asistencia y participación se puede señalar como otro objetivo cumplido satisfactoriamente; pues no se puede obviar que para cualquier persona académica le es de especial interés el contar con la asistencia del grupo completo, pero además, basados en una posición socioconstructivista, lo que más se espera es que esas personas estudiantes tengan el deseo e iniciativa de participar en los distintos espacios que se generan, con el fin de favorecer, deconstruir y analizar el conocimiento. De esta manera, desde la experiencia vivida, se puede afirmar que el estudiantado del curso de PPSO mantuvo una participación sobresaliente en las distintas sesiones sincrónicas programadas a lo largo del semestre, especialmente se resalta el oportuno conocimiento teórico, técnico y actitudinal; además del interés mostrado por incorporar nuevos constructos a su bagaje de conocimientos.

Finalmente, se puede considerar que las modificaciones relacionadas con el incremento de los rubros evaluativos por parte del responsable del curso, genera una mayor participación e interacción dialógica, horizontal y permanente con el colectivo estudiantil; esto se refleja en la expresión de mensajes recibidos de manera directa de estudiantes quienes expresaban que el curso incentivó su amor por la carrera, agradecimientos por el favorecimiento de sus habilidades, destrezas y crecimiento profesional, indicaban que el curso permitió ser un aporte para la realimentación y la unión de grupo, incluso para reconocer las cualidades de sus compañeros y compañeras que nunca habían tenido la oportunidad de conocer; aspectos que se relacionan estrechamente con el aprendizaje significativo; por lo tanto, es recomendable que se pueda tomar en cuenta estos aspectos en el diseño del programa del curso de PPSO 2021 para favorecer una vinculación activa y significativa del académico responsable del curso con el estudiantado.

Si se hace una mirada retrospectiva de todo lo vivido, es meritorio resaltar la capacidad de las personas académicas para la innovación de procesos educativos en la enseñanza superior, no solo en la integración de las herramientas tecnológicas, sino también en el favorecimiento de habilidades personales y profesionales para el estudiantado, desde el trabajo colaborativo y comprometido con su proceso de formación hasta cuando asumían su rol de acompañante, facilitador y mediador de procesos integrado a su realidad contextual y no en el rol pasivo de docente evaluador.

Resalta la importancia de la vinculación de las personas partícipes del proceso de PPSO 2021, debido a que, según lo experimentado, se generan mayores y oportunas acciones de práctica profesional con mayor pertinencia y conocimiento de causa desde la realidad vivenciada durante el 2020.

Idealmente, esta sistematización se puede aprovechar como insumo para la inducción a las experiencias de práctica profesional supervisada; pues se identifica de manera clara y detallada el quehacer en esta, y la importancia de la transformación de los rubros de evaluación del curso, tanto de los evaluados por las personas académicas supervisoras como la persona académica responsable del curso; pues se evidenció una mayor vinculación docente-estudiante en la mediación y desarrollo de este.

Finalmente, la reflexión de mayor relevancia radica en mantener la modalidad de presencialidad remota en la Práctica Profesional Supervisada 2021; pues no sería conveniente exponer al estudiantado a riesgos de salud, traslado de residencia, cambios de trabajo y readaptaciones en el ámbito personal y familiar. Además, desde la experiencia estudiantil y docente, han quedado respaldados los sobresalientes resultados en torno a la aceptación y el aprovechamiento para la construcción del conocimiento desde esta modalidad. 
Como producto del trabajo de evaluación permanente durante la experiencia del curso de PPSO emergieron algunos elementos que, quizás no formaron parte de los objetivos de este estudio, pero se han considerado reflexiones pertinentes y oportunas en la toma de decisiones para favorecer mejoras en los procesos de planificación, ejecución y evaluación de las futuras experiencias de PPSO, a saber:

Las condiciones enfrentadas por atención de la pandemia deben ser registradas y estudiadas por años en la educación latinoamericana, debido a las transformaciones que implicó para el sistema educativo en general para su abordaje. De igual manera, hoy sabemos que las realidades educativas que se conocieron hasta el 2019 nunca volverán a ser las mismas, luego del impacto que han sufrido los procesos de enseñanza y aprendizaje en los diversos escenarios educativos.

Se plantea un reto y un compromiso para el diseño del curso de PPSO 2021; pues es necesario valorar los aprendizajes más sobresalientes de la experiencia vivenciada durante el 2020. Asimismo, comprender que la normalidad se ha transformado a nivel global y debemos garantizar la mejor calidad de la educación superior. Tomar en cuenta las experiencias y las consideraciones enumeradas en el presente documento para valorar la importancia que les asigna el estudiantado y en una modalidad de presencialidad remota, este entorno favorece aspectos de horarios, traslados, recursos, desarrollo de habilidades como el trabajo en equipo, uso de herramientas tecnológicas, organización y gestión de actividades académicas en la modalidad virtual como los webinarios y el reconocimiento a los procesos de actualización profesional.

El cambio de la modalidad presencial a la presencialidad remota impuesta en la Universidad Nacional para la atención a la Emergencia Nacional ante la pandemia provocada por el virus responsable de la COVID-19, implicó una transformación de la vida personal, académica y universitaria, por ejemplo estudiantes de zonas alejadas al cantón central de Heredia debieron regresar a sus casas, y dejaron atrás proyectos marcados por cambios de residencia y adaptación al entorno universitario, algunas personas estudiantes se vieron en la necesidad de buscar opciones laborales o bien, vincularse a las labores propias de la dinámica familiar, con el fin de apoyar en la situación económica y sus compromisos personales, se vincularon con las responsabilidades hogareñas entre muchas otras e incluso emprendieron algunos proyectos.

Todas estas circunstancias descritas en el párrafo anterior deben ser tomadas en cuenta para definir los eventuales procesos de retorno a la modalidad presencial, esta que deberá ser gradual y flexible.

Con los resultados de la presente sistematización se hace necesario extraer argumentos de interés para promover investigaciones relacionadas al tema central, en tres líneas de investigación fundamentalmente; primero, mesurar la desventaja que implica para la población estudiantil que pronto se vinculará al mercado laboral, la limitante de no integrarse a las realidades laborales de manera presencial para conocer esa vida institucional marcada por las interacciones, el entorno, la cultura, las funciones disciplinarias del día a día y las dinámicas propias del mundo del trabajo; segundo, se considera pertinente determinar las virtudes y las ventajas que implica la presencialidad remota que aunque corresponde a una realidad emergente brinda rasgos propios de la virtualidad que deben ser tomadas en cuenta para los diversos procesos de mejora para los diseños o rediseños de carreras de la enseñanza superior. Por último, la tercera línea tiene que ver con el impacto que ha tenido en las instituciones y los escenarios laborales de manera comparativa las experiencias de PPSO mediante la presencialidad y la presencialidad remota para establecer la mejor ruta a seguir en estas. 
CAF-CEPAL. (2020). Las oportunidades de la digitalización en América Latina frente al Covid-19. Recuperado de https://repositorio.cepal.org/bitstream/handle/11362/45360/ OportDigitalizaCovid-19_es.pdf?sequence $=4 \&$ isAllowed $=y$

Camacho, S., Átala, C., Maldonado, G., Álvarez, A., Flores, T., y Santander, T. (2011). Proceso De La Investigación Cualitativa; Epistemología. Recuperado de https://www.uaeh.edu.mx/scige/ boletin/tlahuelilpan/n4/r2.html

Comisión Económica para América Latinay el Caribe (CEPAL)/Organización de Estados Iberoamericanos para la Educación, la Ciencia y la Cultura (OEI), (2020). “Educación, juventud y trabajo: habilidades y competencias necesarias en un contexto cambiante", Documentos de Proyectos (LC/TS.2020/116), Santiago, Comisión Económica para América Latina y el Caribe (CEPAL). Recuperado de https:// repositorio.cepal.org/bitstream/handle/11362/46066/4/S2000522_es.pdf

Decreto Ejecutivo 42227-MP-S. (2020). Declaratoria de Estado de Emergencia Nacional. Recuperado de: http://extwprlegs1.fao.org/docs/pdf/cos194273.pdf

Hodges, C.; Moore, S.; Lockee, B.; Trust, T. y Bond, A. (2020). The Difference Between Emergency Remote Teaching and Online Learning. En Educause Review. Recuperado de https://er.educause. edu/articles/2020/3/the-difference-between-emergency-remote-teaching-and-online-learning

García, F., Corell, A., Abella, V., y Grande, M. (2020) La evaluación online en la educación superior en tiempos de la COVID-19. Education in the Knowledge Society, 21(12). doi:10.14201/eks.23013

González, K. (2020). Del aula presencial a la remota y de regreso: la enseñanza en situaciones de pandemia e incertidumbre. Revista Panamericana De Pedagogía, 31(2021), 10-25. doi: E-ISSN2594-2190.

Moreira y Delgadillo (Enero-marzo 2014). La virtualidad en los procesos educativos: reflexiones teóricas sobre su implementación. Tecnología en Marcha, 28(1):121-129. Recuperado de https:// www.scielo.sa.cr/pdf/tem/v28n1/0379-3982-tem-28-01-00121.pdf

Moreno, T. (Abril-junio 2009). La Evaluación Del Aprendizaje En La Universidad. Tensiones, Contradicciones y Desafíos. RMIE, 14(41):563-591. Recuperado de: http://www.scielo.org.mx/pdf/ rmie/v14n41/v14n41a10.pdf

Peña, C. y Vargas, L. (2020). La práctica profesional, retos y desafíos para el apoyo organizacional. Bogotá: Corporación Universitaria Minuto de Dios. UNIMINUTO, 2020. Recuperado de https:// repository.uniminuto.edu/bitstream/handle/10656/11233/Libro_Internas-La\%20practica\%20 profesional\%2c\%20retos\%20y\%20desafios\%20para\%20el\%20apoyo\%20organizacional_2020. pdf?sequence $=1$ \&isAllowed $=\mathrm{y}$

Salgado, E. (2006). Manual de Docencia Universitaria. Introducción al Constructivismo en la Educación Superior.EditorialULACIT. Recuperado dehttps://www.researchgate.net/publication/234672807_ Manual_de_Docencia_Universitaria_Introduccion_al_Constructivismo_en_la_Educacion_ Superior

Sánchez, A. (Febrero-mayo 2010). El artículo sistematización de experiencias: construcción de sentido desde una perspectiva crítica. Revista Virtual Universidad Católica del Norte, 29:1-7. Recuperado de https://www.redalyc.org/pdf/1942/194214466001.pdf

Sánchez, J. (2003). La Docencia Universitaria. Para un manifiesto antipedagógico. Quito, Ecuador: Ediciones Abya-Yala. Recuperado de: https://digitalrepository.unm.edu/cgi/viewcontent. cgi?article=1136\&context=abya_yala

Universidad Nacional. (2017). Normativa Institucional. Publicado en La Gaceta Diario Oficial n. ${ }^{\circ}$ 7-2017 del 15 de junio de 2017. Recuperado de: https://documentos.una.ac.cr/bitstream/handle/ unadocs/8213/UNAGACETA72017_firmado.pdf?sequence=1\&isAllowed=y 
Consejo Académico-Universidad Nacional. (2020). Acta de la Sesión Ordinaria N.o 8-2020. Recuperado de: https://www.consaca.una.ac.cr/index.php/documentos/category/60-a2020

Zielińsk, K., Jaruga, A., Hofmann, R., Marinova, T., Plewczynski, M., Kerler, M. (2012). Webinar methodology. Webinar2learn. Recuperado de: https://ec.europa.eu/programmes/erasmus-plus/ project-result-content/9dfd6607-60f7-4b3d-870e-6be51d4b15df/metodyka_EN_FINAL.pdf 